Brigitte Glaser

\title{
,In the East my pleasure lies': Zur Darstellung und Rolle des Exotischen in Shakespeares Dramen
}

Das während der Frühen Neuzeit plötzlich wachsende Interesse am Exotischen, am Seltsamen und völlig Anderen lässt sich am besten aus dem historischen Kontext heraus erklären und verstehen. So gab es im 16. und 17. Jahrhundert zahlreiche Forschungsexpeditionen in geographisch weit entlegene Gegenden der Welt sowie Berichte über fremde Länder und bisher unbekannte Völker. ${ }^{45}$ Das Lesen dieser Berichte, wie etwa Richard Hakluyts Principal Navigations oder von Reiseerzählungen, wie sie Samuel Purchas sammelte, ${ }^{46}$ gewann an Popularität, und das zu

45 In diesen Zeitraum fallen die Gründung der East India Company (1567), Sir Walter Raleighs Reisen nach Virginia und Venezuela, sowie Sir Francis Drakes zu den westindischen Inseln, aber auch diverse Expeditionen der Entdecker Martin Frobisher, William Baffin und Henry Hudson in die Arktis, die zum Ziel hatten, eine „Nordwestpassage“ ausfindig zu machen.

46 Richard Hakluyt veröffentlichte im Jahr 1589 die erste Sammlung von Briefen und Reiseberichten unter dem Titel The Principal Navigations, Voyages and Discoveries of the English. Eine weitere, überarbeitete Fassung erschien in drei Bänden zwischen 1598 und 1600. Samuel Purchas publizierte in seinem Werk Samuel Purchas, His Pilgrimage (1613) bereits bekannte, jedoch modifizierte, mit eigenen Interpretationen und Theorien versehene Berichte von Seefahrten und englischen Expeditionen, wie auch von religiösen Bräuchen in fernen Ländern. 
einer Zeit, als England sich bedroht fühlte und wiederholt die Angriffe fremder Mächte fürchtete (nicht ohne Grund, wie das Erscheinen der spanischen Armada im Jahr 1588 zeigte). Auf diesem Hintergrund lassen sich auch die unterschiedlichen Wahrnehmungen des Exotischen verstehen: einerseits wurden Reiseberichte und Beschreibungen neuer Entdeckungen mit Interesse und Neugier aufgenommen, andererseits riefen gerade die Ängste vor einer erneuten Invasion ausländischer Mächte bei Engländern eine gewisse Skepsis und Vorsicht, wenn nicht gar eine ablehnende Haltung gegenüber anderen Völkern hervor. Das Zusammentreffen mit dem Anderen, entweder direkt in der Form von persönlichen Begegnungen oder indirekt durch dessen Rezeption in diversen Diskursen, erforderte aber auch eine Auseinandersetzung mit sich selbst, wie gerade jenen Texten der Epoche zu entnehmen ist, die Fragen der Identität zum Thema haben, eine Erforschung des Selbst zum Gegenstand machen und eine Tendenz zur Selbstdefinition erkennen lassen. So zeigt etwa die berühmte Aussage des Earl of Rochester - „In my dear self I center everything“" (Wilmot 113) [, auf mein eigenes, mir so vertrautes Selbst konzentriere ich alles" (Ü: BG)] - in welchem Ausmaß die Erforschung des Individuums inzwischen ins Zentrum aller Aufmerksamkeit gerückt war. Jedoch fand dieser Prozess der Selbsterforschung häufig in Abgrenzung des Selbst zum Anderen statt und zog eine Verteilung positiver und negativer Charakteristika nach sich, wobei gerade die andere, fremde Seite mit einer Reihe diskriminierender Stereotypen versehen wurde.

Die französische Psychologin und Philosophin Julia Kristeva hat die Schnittpunkte zwischen der Vorstellung des Anderen und einer kolonialen Sichtweise erforscht und in Fremde sind wir uns selbst (2001) [Étrangers à nous-mêmes (1988)] aufgezeigt, dass die Idee des „Fremden in uns“ dazu führte, Orientalisches oder Afrikanisches mit jenen Elementen westlicher Gesellschaften in Verbindung zu bringen, die als fremdartig und nichtkonform wahrgenommen wurden, etwa mit Straftätern, Geisteskranken, Frauen und Armen (Kristeva 20). Genau diese Bilder des Anderen wurden dann zusammen mit dem Anderen innerhalb der eigenen Gesellschaft an den Rand gedrängt. Kristeva erläutert, dass Vorstellungen vom Orient und von Afrika unbewusst mit dem Anderssein assoziiert und zu Bildern des eigenen „verbotenen“ oder „verlorenen Selbst“ wurden, also zu unterdrückten Spiegelbildern von Europa (Kristeva 146, 200-201). Auch in Shakespeares Dramen läßt sich die Verknüpfung von Anderssein und Normabweichung nachweisen und zwar anhand von immer wieder auftretenden Diskursen zu Wahnsinn, Gottlosigkeit, Unmoral, Unordnung, Deformität, Minderwertigkeit und Gewalt, die im Zusammenhang mit exotisch, seltsam oder nichtkonform erscheinenden Personen verwendet werden. Zugleich aber verknüpft der Autor die Darstellung des Exotischen und Fremden mit Anklängen an die Erotik, und dabei vor allem mit der Sehnsucht nach dem Verlorenen, häufig projiziert in der Form eines weiblichen Körpers und eingebettet in Diskurse um Verlangen und Verlust. Da sich aber, wie der Literatur- und Kulturkritiker Edward Said in seiner einflussreichen 
Studie Orientalism schreibt, das europäische Kolonialprojekt auf die Idee einer europäischen Identität stützt, die als allen anderen nichteuropäischen Völkern und Kulturen überlegen gesehen wird (Said 1-28), ${ }^{47}$ kann auch die Konstellation um Verlangen und Verlust nicht eindeutig repräsentiert werden: das Exotisch-Fremde wird zum Objekt, das sowohl eine Anziehungkraft ausübt als auch Ängste hervorruft. Ein gutes Beispiel für den Umgang mit dieser widersprüchlichen Wirkung des Anderen ist etwa die Repräsentation des Körpers in Antony and Cleopatra: so zeigt der Dramatiker, dass Cleopatras Körper Antony in seinem Handeln bestimmt, zugleich aber auch bei seinen Anhängern Ängste und Zweifel bezüglich Antonys Männlichkeit und Autorität hervorruft.

Ziel der folgenden Ausführungen wird sein, anhand einer Dramenauswahl, nämlich in chronologischer Reihenfolge The Merchant of Venice, Othello, Antony and Cleopatra und The Tempest, 48 die komplexe Beziehung zwischen Selbst und Anderem zu untersuchen, und zwar in ihren Repräsentationsformen und -funktionen. Der Fokus wird dabei zum einen auf der semantischen Konstruktion dieser Beziehung als kontrastierendes Paar liegen, bei dem die eine Seite als normativ, die andere als davon abweichend oder unzulänglich präsentiert wird. Zum anderen soll als Hintergrund der Dramen die zeitgenössische englische Gesellschaft miteinbezogen werden, in dem Sinne, dass das eine oder andere von Shakespeares Stücken den zuschauenden Engländern ermöglichte, aufgestaute und unterdrückte Ängste loszuwerden und dadurch an Selbstbewusstsein (auch im Sinne von nationalem Bewusstsein) zu gewinnen. Am Anfang soll eine Komödie stehen, die wegen ihrer märchenhaften Aspekte und der Verlagerung des Handlungsorts nach Italien beim englischen Publikum Anklang fand, zugleich aber in ihren xenophobischen Aspekten gewisse stereotype Darstellungsweisen des Anderen bestätigte.

\section{The Merchant of Venice}

In diesem Stück, das wohl in den Jahren 1596-98 entstanden war, bringt Shakespeare zwei Figuren mit dem Exotischen und Fremden in Verbindung. Eine davon ist eine Nebenfigur, nämlich ein untypischer, da nicht-schurkenhafter Bühnenmohr. Die andere aber ist eine Hauptfigur, die als gnadenlos hart und nachtragend dargestellt und als ,inexecrable dog“ (IV.i.128) [,unbarmherz'ger

47 Explizit schreibt Said dazu: „Orientalism is never far from [...] the idea of Europe, a collective notion identifying 'us' Europeans as against all 'those' non-Europeans and indeed it can be argued that the major component in European culture is precisely what made that culture hegemonic both in and outside Europe: the idea of European identity as a superior one in comparison with all the non-European peoples and cultures." (Said 7)

48 Für die englischen Zitate aus den Shakespeare-Dramen wird die Arden-Edition verwendet, für die deutsche Fassung dieser Zitate die von Levin Schücking 1955 wieder herausgegebene Schlegel-Tieck-Übersetzung (siehe Bibliographie). 
Hund“ (Bd. II, 532)] bezeichnet wird. Was beide gemeinsam haben, ist das Merkmal der Gottlosigkeit (Rosen 67-79).

Die erste Figur, der Prinz von Marocco, ein Werbender um Portias Hand, ist zugleich einer jener vielen Fremden, die das kosmopolitische Venedig besuchten oder dort vorübergehend lebten, in jener Stadt, die sich im 16. Jahrhundert zum Zentrum des Welthandels entwickelt hatte. Er ist darin jenen vereinzelten Mauren nicht unähnlich, die im zeitgenössischen London einen temporären Aufenthaltsort gefunden hatten (Jones 12). Wie auch Othello ist sich der Prinz von Marocco seines Andersseins, d.h. seiner Anstoß erregenden äußeren Erscheinung bewusst. Er betritt deshalb den Wettbewerb um Portia mit den Worten

Mislike me not for my complexion

The shadowed livery of the burnished sun,

To whom I am a neighbour and near bread

[Verschmähet mich um meine Farbe nicht,

Die schattige Livrei der lichten Sonne,

Die mich als nahen Nachbar hat gepflegt.

(Bd. II, 492)]

Die junge Frau aber scheint ihre Entscheidung schon vor seiner Wahl eines der drei Kästchen getroffen zu haben, denn sie vergleicht sein Aussehen mit dem des Teufels und mockiert sich über seine vermeintliche Gottlosigkeit: „if he have the condition of a saint, and the complexion of a devil, I had rather he should shrive me than wive me“ (I.ii.123-25) [,,Hat er das Gemüt eines Heiligen und das Geblüt eines Teufels, so wollte ich lieber, er weihte mich, als er freite mich" (Bd. II, 486/7)]. In der Erwartung, dass ihr künftiger Ehemann ein gottesfürchtiger Europäer sein würde, lehnt sie den Prinzen von Anfang an wegen seiner Hautfarbe und seines Glaubens ab. Mit des Prinzen Wahl des goldenen Kästchens spielt Shakespeare zudem auf orientalische Hybris und Extravaganz an und macht den fremden Adeligen vor den Augen des zeitgenössischen Bühnenpublikums zu einem (be)gierigen Mohren, der zurecht ausgeschlossen und verbannt wird, da er sich nicht mäßigen und für das bescheidenere Bleikästchen entscheiden kann.

Shakespeare behandelt das Thema der Gottlosigkeit als Ursache für Unordnung noch weit ausführlicher in der Beschreibung des Shylock, jenes anderen Fremden, der mit dem Teufel assoziiert wird, nun aber nicht wegen der Hautfarbe, sondern wegen des alten Fluchs, der mit der Kreuzigung Jesu entstand. Durchwegs als „der Jude“ bezeichnet, fungiert Shylock weniger als Individuum denn als Repräsentant eines Volks. Die Art, wie man mit ihm umgeht, erinnerte das zeitgenössische Publikum sehr wahrscheinlich an den jüdischen Arzt Rodrigo Lopez, den man 1594 (also wenige Jahre vor der Entstehung des Stücks) zum Tode verurteilt hatte, da er angeblich an einer Verschwörung beteiligt gewesen sein soll, die die Vergiftung Königin Elisabeths zum Ziel hatte. Im Gegensatz zu 
seiner Tochter Jessica, die mit ihrer Heirat des Lorenzo zum Christentum konvertiert, erweist sich Shylock als hoffnungslos verloren, was seinen Glauben anbelangt und wird deshalb wiederholt als „,the dog Jew“ (II.viii.14) [„,der Hund von Juden“ (Bd. II, 506)] und „,cut-throat dog“ (I.iii.114) [„Bluthund“ (Bd. II, 489)] tituliert. Trotz seiner Bitten, ihn doch wie einen Menschen zu behandeln, - „Hath not a Jew hands, organs, / dimensions, senses, affections, passions“ (III.i.53/54) [,,hat nicht ein Jude Hände, Gliedmaßen, Werkzeuge, Sinne, Neigungen, Leidenschaften?" (Bd. II, 512)] - wird er im Laufe der Handlungsentwicklung zur Quelle des Bösen, das überwunden und beseitigt werden muss. Die ihm auferlegte schwere Strafe und sein Ausschluss aus der venezianischen Gesellschaft hatten vermutlich die Funktion, die Zuschauer in ihrem Gefühl ethnischer und religiöser Überlegenheit zu bestärken und ihnen im Umgang mit Ängsten vor einer Invasion fremder Mächte zu helfen. Den hier als Nebenfigur eingeführten Mauren sollte Shakespeare einige Jahre später weiterentwickeln, ihm charakterbezogene Tiefe geben und ihn vor allem auch in seiner exotischen Dimension noch weit sichtbarer markieren.

\section{Othello}

Ein „schwarzer Mann“ steht im Zentrum von Shakespeares Othello, einem Stück, das zwischen 1602 und 1604 entstand und im Jahre 1604 uraufgeführt wurde. Othellos Anderssein manifestiert sich nicht nur in den Aspekten seiner äußeren Erscheinung, sondern auch in seinem Glaubens und seinem Verhalten. Während seine dunkle Haut Assoziationen mit dem Bösen evoziert und ihn damit zu einem potentiellen Monster macht, impliziert sein Glaube Gottlosigkeit und Unmoral, denn Othello ist ein mauretanischer Muslim. Zudem bestärkt sein Verhalten im Laufe der Handlung des Publikums anfängliche Annahmen über ihn: Othello scheint sich allmählich von einem edlen Wilden zu einem gefährlichen Wahnsinnigen zu wandeln, einem, von dem die Gefahr ausgeht, dass er Unordnung über den venezianischen Staat bringt.

Die Wirkung einer Figur wie Othello auf zeitgenössische Zuschauer lässt sich am besten im historischen Kontext verstehen: vor dem 16. Jahrhundert hatte England im Gegensatz zu den mediterranen Ländern kaum Kontakt mit aus Asien oder Afrika stammenden Menschen unterschiedlicher Hautfarbe gehabt. Zwar war während des elisabethanischen Zeitalters die Anzahl der Mauren in London gestiegen, diese waren aber überwiegend phänotypisch wenig auffällig. Othello aber ist als Schwarzer konzipiert (Jones 23) und deshalb Furcht einflößend. Mangels Erfahrung neigte das Bühnenpublikum im England der Renaissance noch dazu, über Wesen aus fernen Ländern schockiert zu sein und Bilder des Seltsamen und Fremden lange Zeit in Erinnerung zu behalten. Von Bedeutung in diesem Zusammenhang ist auch, dass trotz der Existenz authentischer, auf tatsächlich stattgefundenen Reisen basierender Information eben gerade legendenhafte Vorstel- 
lungen einen wichtigen Bestandteil der populären Imagination ausmachten und eine reichhaltige Mischung aus Fakten, Mythen und Phantasie etwa über das afrikanische Andere bildeten (Jones 1-26). Othello selbst wählt im ersten Akt für seine afrikanischen Erzählungen Geschichten von Kannibalen und gibt damit jener stereotypisierenden europäischen Sichtweise Ausdruck, die auf der Imagination, nicht aber auf Fakten beruhte. Bereits zu diesem Zeitpunkt ist er als fiktionales Anderes konstruiert, zeigt sich aber als sensibilisiert für die europäische Perspektive. In der Tat scheint er also zu wissen, welche Geschichten die Europäer am liebsten hören (Loomba, 2002, 91-105).

Othellos Transformation vom Guten zum Bösen wird von Shakespeare explizit mit Sexualität und orientalischer Sinnlichkeit in Verbindung gebracht. Zwar beginnt die Beziehung zwischen Othello, dem „black ram“ (I.i.88) [also dem „schwarzen Schafbock“ (Bd. V, 8)] und Desdemona, dem „white ewe“ (I.i.88) [d.h. dem ,weißen Lämmchen“ (Bd. V, 8)] in Venedig, wird aber bald darauf nach Zypern verlegt, das zur damaligen Zeit als Insel des Ostens bekannt war. Dass der Osten im Stück eine wichtige Rolle spielt, geht auch daraus hervor, dass Desdemonas Verführung gerade durch jene exotischen Geschichten gefördert wird, die ihr Othello erzählt, Geschichten, die sie in ferne Länder tragen und ihr fremde Völker und wundersame Verhaltensweisen vor Augen führen, sie aber auch mit jenen heroischen Taten vertraut machen, die Othello dort vollbracht hat. Später beschreibt Othello die Wirkung seines Geschichtenerzählens folgendermaßen: „She lov'd me for the dangers I had pass'd, / And I lov'd her that she did pity them" (I.iii.167/68) [,,sie liebte mich, weil ich Gefahr bestand; / Ich liebte sie um ihres Mitleids willen:“ (Bd. V, 18)]. Seiner Anziehungskraft, aber auch ihrem eigenen Verlangen nachgebend verletzt Desdemona die Anweisungen des Vaters sowie die Erwartungen der Gesellschaft, als sie sich mit einem dunkelhäutigen Mann einlässt. Von Anfang an spricht Othellos äußere Erscheinung gegen ihn und führt dazu, dass ihm, dem für seine militärischen Errungenschaften hoch Ausgezeichneten, Zweifel und Argwohn entgegenschlagen. ${ }^{49} \mathrm{Da}$ bei den Elisabethanern der prototypische Bühnenmohr im Rahmen der üblichen afrikanistischen Diskurse mit der Neigung zu Eifersucht assoziiert wurde und da zudem zu jener Zeit eine Verbindung zwischen einem farbigen Mann und einer weißen Frau geradezu undenkbar war, folgt der Handlungsverlauf einer vorbestimmten Logik und endet im Scheitern dieser Beziehung (D’Amico 177-195 sowie Cohwig 7-14). Interessanterweise entwirft Shakespeare aber einen Mohren, der die gegen ihn vorgebrach-

49 So vermutet Desdemonas Vater Brabantio, Othello habe seine Tochter durch „spells and medicines“ (I.iii.61) [„,Hexenkünste und Quacksalbergetränke“ (Bd. V, 16)] verhext, denn sonst wäre es für sie nicht möglich gewesen, ,,in spite of nature, / Of years, of country, credit, everything, / To fall in love with what she fear'd to look on“ (I.iii.96-98) [,,trotz Natur / Und Jugend, Vaterland und Stand und allem, / Das [zu] lieben, was ihr Grauen schuf zu sehn" (Bd. V, 16/17)]. Desdemona jedoch betont, dass für sie die Überwindung des Äußeren durch den Blick auf Othellos innere Werte möglich war: „I saw Othello's visage in his mind“ (I.iii.252) [„Mir war Othellos Antlitz sein Gemüt“ (Bd. V, 21)]. 
ten Vorurteile bereits internalisiert hat und den das Wissen um sein Anderssein zu Schuldgefühlen und schließlich zu Mord und Selbstmord treibt. Shakespeare zeigt auf diese Weise, dass Othello doch noch zu dem wird, was er von Anfang an zu sein scheint, nämlich ein wahnsinniger und mörderischer Außenseiter. Und noch einmal, nach dem Mord an Desdemona und kurz bevor er seinem eigenen Leben ein Ende setzt, erzählt Othello eine orientalische Geschichte, in deren Mittelpunkt dieses Mal ein tragischer Held steht: dieser vermochte den wertvollen Schatz, den er einst hatte, nicht ausreichend zu würdigen und musste sich deshalb dem unehrenvollen Tod eines östlichen Ungläubigen unterziehen. Es ist dies die Geschichte

von einem, dessen Hand

Dem niedern Inder gleich, die Perle wegwarf,

Mehr wert als all sein Volk; des überwundnes Auge

Sonst nicht gewöhnt zu schmelzen, sich ergeußt

In Tränen, wie Arabiens Bäume tau'n

Von heilungskräft'gem Balsam - 50

(Bd. V, 97)

Und Othello fährt fort:

- schreibt das alles;

Und fügt hinzu: daß in Aleppo, wo

Ein gift'ger Türk' in hohem Turban einst

'nen Venetianer schlug und schalt den Staat, -

Ich den beschnittnen Hund am Hals ergriff

Und traf ihn - so!51

(Bd. V, 97)

Und er ersticht sich mit seinem Dolch.

Gerade diese Rechtfertigung des Othello im letzten Akt, in der er Bilder des „niedern Inder“ [,,base Indian“] und des ,gift'gen Türk " [,turban’d Turk“] auf sich selbst bezieht, während er der toten Desdemona metaphorisch die Rolle der bisher wenig geschätzten Perle zuschreibt, mag bei den zeitgenössischen Zuschauern vorgefertigte Annahmen über den Mohren bestärkt und zu jener kathartischen Wirkung geführt haben, die aus der Zustimmung zu Othellos später Einsicht und seinem selbstbewussten Freitod rührte.

Während im Merchant of Venice und in Othello exotische Schauplätze nur andeutungsweise zum Einsatz kommen, fungieren sie in den beiden nachfolgenden Stücken als zentrale Elemente, die gerade auch die Charakterisierung von Frem-

50 „,[of] one whose hand, / Like the base Indian, threw a pearl away, / Richer than all his tribe: of one whose subdued eyes, / Albeit unused to the melting mood, / Drops tears as fast as the Arabian trees / Their medicinal gum"(Othello, V.ii.347-52).

51, ,...] set you down this, / And say besides, that in Aleppo once, / Where a malignant and a turban'd Turk / Beat a Venetian, and traduc'd the state, / I took by the throat the circumcised dog, / And smote him thus.“ (Othello, V.ii.352-57) 
dem und Vertrautem als Gegensatzpaar unterstützen. Vor allem in ihrer Assoziation mit Magie bilden sie zudem den Hintergrund zur Exotisierung einer jeweils zentralen Figur.

\section{Antony and Cleopatra}

In Antony and Cleopatra, einem „Roman play“, das Shakespeare wohl in den Jahren 1606/7 schrieb, re-konfiguriert der englische Dramatiker den konfliktbeladenen Gegensatz von Selbst und Anderem in der Beziehung der beiden Protagonisten und gestaltet ihn nun ausgehend vom weiblichen Körper als Zentrum der Anziehung, aber auch Zerstörung. Cleopatra, und dabei vor allem ihr Körper, ist zugleich ein Objekt männlicher Phantasien und ein Symbol für den als weiblich konnotierten Orient, denn sie führt Antony auf Irrwege und zerstört dabei seine virtus, d.h. seine Maskulinität im Sinne römischer Denkvorstellungen (Loomba, 1989, 124-130). Shakespeare assoziiert Cleopatras Weiblichkeit mit Hexenkunst und deutet damit an, dass Antony dieser machtvollen Verlockung nicht entkommen kann. Antonys Rivale Pompey beschreibt diese Gefahr wie folgt:

\section{Doch Liebreiz würze}

Der üpp'gen Cleopatra Zauberlippen,

Zauber erhöh' die Schönheit, Wollust beide;

Den Schwelger bind' ein Heer von Festgelagen,

Sein Hirn umnebelnd; epikur'sche Köche

Schärfen mit kräftig neuen Brühn die Eßlust,

Daß Schlaf und Schwelgen seinen Ruhm vertagen,

Bis zur Betäubung Lethes. 52

Obgleich sich die als rational gezeichneten Römer der Gefahren, die von Ägypten ausgehen, bewusst zu sein scheinen, können sie sich dem Zauber und der Anziehungskraft des Fremden und Exotischen kaum entziehen, wie aus der nachfolgenden Beschreibung des Enobarbus, eines Gefolgsmanns des Antony, hervorgeht. Seine Beschreibung Ägyptens als ein phantastischer Ort, der ein Fest der Sinne ermöglicht, ist ein Gemeinplatz orientalistischer Repräsentation und impliziert den Orient als verlorenes Paradies, unerreichbar und verboten, als das, was wir uns wünschen, doch zu unterdrücken gezwungen sind:

Die Bark', in der sie saß, ein Feuerthron,

Brannt' auf dem Strom: getriebnes Gold der Spiegel,

52 „But all the charms of love, / Salt Cleopatra, soften thy waned lip! / Let witchcraft join with beauty, lust with both; / Tie up the libertine in a field of feasts; / Keep his brain fuming. Epicurean cooks / Sharpen with cloyless sauce his appetite / That sleep and feeding may prorogue his honour / Even till a Lethe'd dullness." (Antony and Cleopatra, II.i.20-27) 
Die Purpursegel duftend, daß der Wind Entzückt nachzog; die Ruder waren Silber,

Die nach der Flöten Ton Takt hielten, daß

Das Wasser, wie sie's trafen, schneller strömte, Verliebt in ihren Schlag. Doch sie nun selbst, --

Zum Bettler wird Bezeichnung: sie lag da

In ihrem Zelt, das ganz aus Gold gewirkt,

Noch farbenstrahlender, als jene Venus,

Wo die Natur der Malerei erliegt.

Zu beiden Seiten ihr holdsel'ge Knaben,

Mit Wangengrübchen, wie Cupido lächelnd,

Mit bunten Fächern, deren Wehn durchglühte

(So schien's) die zarten Wangen, die sie kühlten;

Anzündend statt zu löschen.

$[\ldots]$

Die Dienerinnen, wie die Nereiden,

Spannten, Sirenen gleich, nach ihr die Blicke,

Und Schmuck ward jede Beugung; eine Meerfrau

Lenkte das Steuer; seidnes Tauwerk schwoll

Dem Druck so blumenweicher Händ' entgegen

Die frisch den Dienst versahn. Der Bark' entströmend

Betäubt' ein würz'ger Wohlgeruch die Sinne

Der nahen Uferdämme. ${ }^{53}$

$$
\text { (Bd. V, 191/2) }
$$

Obwohl diese Beschreibung einerseits männliche Phantasien von begehrenswerten, sinnebetörenden Schauplätzen zu evozieren vermag, sind die hier entworfenen Bilder doch überwiegend der populären Imagination entnommen. Sie konnten um etwa die gleiche Zeit auch in Raleighs Schilderung des legendären El Dorado gefunden werden und würden später wieder in den beliebten Erzählungen der Arabian Nights, also den Geschichten aus 1001 Nacht anzutreffen sein.

53 „The barge she sat in, like a burnished throne, / Burned on the water; the poop was beaten gold; / Purple the sails, and so perfumed that / The winds were love-sick with them; the oars were silver, / Which to the tune of flutes kept stroke, and made / The water which they beat to follow faster, / As amorous of their strokes. For her own person, / It beggared all description: she did lie / In her pavilion, cloth-of-gold of tissue, / O'erpicturing that Venus where we see / The fancy outwork nature. On each side her / Stood pretty dimpled boys, like smiling cupids, / With divers-coloured fans, whose wind did seem / To glow the delicate cheeks which they did cool / And what they undid did. / [...] / Her gentlewomen, like the Nereides, / So many mermaids, tended her i' th' eyes, / And made their bends adornings. At the helm / A seeming mermaid steers. The silken tackle / Swell with the touches of those flower-soft hands / That yarely frame the office. From the barge / A strange invisible perfume hits the sense / Of the adjacent wharfs." (Antony and Cleopatra, II.ii.199-221) 
Die Darstellung der Cleopatra als das zentrale Andere im Stück ist jedoch auch zweideutig, denn diese so verlockende Frau ist dezidiert negativ konnotiert. Cleopatra, die schließlich den Untergang des Antony herbeiführen wird, wird im Laufe der Handlung als Hure, Zigeunerin, Monster, lüsterne Verführerin, wollüstige Stute und „Schlange des Nils“ (Bd. V, 182) bezeichnet, denn aus der Sicht der Römer setzt sie ihren Körper zur Realisierung ihrer politischen Ziele ein. Im Gegensatz zu Elisabeth I., die häufig mit vornehmer Jungfräulichkeit assoziiert wurde, erscheint Cleopatra in ihrer Herrscherrolle als unakzeptabel, wird sie doch mit männlicher Aggressivität in Verbindung gebracht. Cleopatras Körper, zugleich Objekt der Begierde und Quelle aller Ängste, wird von Shakespeare zudem als, wie es der Zeit entsprach, weiblich konnotiertes Territorium dargestellt, das männlicher Kontrolle zu unterwerfen ist. Wir finden ähnliche Vorstellungen etwa in Sir Walter Raleighs Discoverie of Guiana (1596) oder aber in John Donnes Gedicht „Elegy XIX: To His Mistress Going to Bed“ (1633), in dem der Sprecher die Geliebte als „O, my America! my New-found-land!“ (Donne, Zeile 27) adressiert und dann ihren Körper zu entdecken und zu besitzen sucht (Waddington 289-291). In Shakespeares Stück muss dieser Körper ebenso erobert und kontrolliert werden, auch wenn der Preis dafür die Vernichtung der beiden Liebenden und die Wiederherstellung europäischer, hier römischer Vorstellungen von sozialer Ordnung ist. Die hier bereits angedeuteten kolonialen Prozesse und Strukturen, die üblicherweise die Unterwerfung oder gar Vernichtung anderer, als potentiell subversiv oder als minderwertig gesehener Gegner zum Ziel haben, werden zur zentralen Thematik des oft als Romanze bezeichneten abschließenden Stücks des Shakespeareschen Cuvres.

\section{The Tempest}

Dieses letzte, wohl um 1611 entstandene Drama Shakespeares zeigt bestens, wie koloniale Imagination funktioniert, denn in ihm treten zwei Aspekte hervor, die immer wieder in kolonialen Diskursen anzutreffen sind: zum einen jener Prozess, der das Gegenüber zu einem Anderen macht und zweitens, jene für Machtbeziehungen so notwendige Spannung zwischen Subversion und Kontrolle derselben (Mannoni 105-108; Demaray 110-134). In The Tempest befinden wir uns nun auf einer geographisch nicht näher definierten Insel, die höchst wahrscheinlich irgendwo zwischen Neapel und Tunis gelegen ist, obwohl einige Wissenschaftler sie auch in der Karibik verorten möchten und sie deshalb im Kontext von Diskursen über die „Neue Welt“ analysieren. Es handelt sich um jene Insel, die der Einheimische Caliban zu besitzen glaubt, die ihm aber genommen wurde, als die Kolonisatoren ihn zum Untertan machten. Um ihre weitere Vorgehensweise zu rechtfertigen, konstruieren die Neuankömmlinge Caliban als das widerspenstige und barbarische Andere und deshalb auch als vermeintliche Quelle der Unordnung und Gewalt. Caliban wird zum Rezipienten ihrer Verdächtigungen und ihrer Be- 
mühungen, ein Gegenbild von sich selbst zu entwerfen. Aus der Perspektive der Zuschauer gesehen wird Caliban zudem zum Hauptobjekt, auf das jene Annahmen projiziert werden, die auch die zeitgenössischen Engländer im Laufe ihrer Begegenungen mit Vertretern fremder Länder entwickelten und die, wie Kristeva es ausdrückte, „kulturelle Angst“ hervorriefen (Kristeva 161).

Als die Reisenden in ferne Länder auf der Insel ankommen, bringen sie Mythen mit sich sowie einschlägige Vorstellungen vom Anderen. Ein Beispiel dafür ist Gonzalos Bemerkung zu befremdlicher Missbildung und Entstelltheit, wie man sie an weit entlegenen Orten finden mag:

\section{In unsrer Jugend}

Wer glaubte wohl, es gebe Bergbewohner,

Mit Wammen so wie Stier'n, an deren Hals

Ein Fleischsack hing?? Es gebe Leute, denen

Der Kopf im Busen säße? als wovon

Ein jeder, der sich fünf zu eins versichert,

Gewißheit gibt. ${ }^{54}$

$$
\text { (Bd. VI, 135) }
$$

Obwohl das Stück nach Ansicht einiger in der Neuen Welt spielt, hatte Shakespeare die darin verwendeten Vorstellungen vom Anderssein bereits im früheren Drama Othello benutzt, und zwar in den afrikanischen Erzählungen, in denen „Anthropophagen, Völker[n], deren Kopf / Wächst unter ihrer Schulter“ (Bd. V, 18) [,The Anthropophagi, and men whose heads / Do grow beneath their shoulders“ (Othello, I.iii.144-45)] vorkommen. Dieses Beispiel zeigt die Übertragbarkeit prägnanter Metaphern und Bilder und konstituiert einen wichtigen Bestandteil des Prozesses, das Gegenüber zum Anderen zu machen (Boehmer 12-59).

Doch zurück zu Gonzalo und seinen Freunden, ihrer Kenntnis der alten Geschichten und Mythen, und ihrem Zusammentreffen mit dem Exotischen und Fremden. Einige Aspekte dieser Mythen glauben sie nun im seltsam anzusehenden Caliban wiederzuerkennen. Deshalb bezeichnen sie ihn als „monster“ (II.ii.30), ,a strange fish“ (II.ii.27), a „devil“ (IV.i.188), a „hag-seed“ (I.ii.365), und „a freckled whelp hag-born“ (I.ii.283) [,Monster“, „ein seltsamer Fisch“ (Bd. VI, 122), ,Teufel“, „Hexenbrut“ (Bd. VI, 107) und „ein scheckig Wechselbalg“ (Bd. VI, 105)] und bringen bald seine äußere Erscheinung mit seinem Verhalten in Verbindung: „Er ist so ungeschlacht in seinen Sitten / Als von Gestalt“ (Bd. VI, 153) ${ }^{55}$. Neben der Figur des Caliban steuern aber auch die Hexe Sycorax, Calibans Mutter aus Algier, sowie das Geisterwesen Ariel zum Entstehen der exotisch-

54 „When we were boys, / Who would believe that there were mountaineers / Dew-lapp'd like bulls, whose throats had hanging at 'em / Wallets of flesh? or that there were such men / Whose heads stood in their breasts? which now we find / Each putter-out of five for one will bring us / Good warrant of." (The Tempest, III.iii.43-49)

55 „He is as disproportioned in his manners / As in his shape“ (V.i.291-92). 
fremden Atmosphäre im Stück bei. Prospero, der selbsternannte, neue Herrscher über die Insel, versucht Kontrolle zu bekommen, indem er die ursprünglichen Bewohner gegeneinander ausspielt: da er Caliban des Widerstands und der Rebellion verdächtigt, sei es in Form einer Attacke auf seine unschuldige Tochter Miranda oder aber in Form von Komplottplänen gegen ihn, führt Prospero ein System der Überwachung, Unterdrückung und Bestrafung ein und betraut Ariel als Wächter und Handlungsausführender mit dessen Umsetzung. Dabei ist vor allem interessant, aber nicht überraschend, dass Prosperos wachsendem Argwohn bezüglich des einheimischen Calibans Anstrengungen vorangegangen waren, diesen $\mathrm{zu}$,zivilisieren“, indem er ihm eine fremde Sprache und Kultur nahezubringen versuchte. Diese Betonung des eigenen, überlegenen linguistischen und kulturellen Hintergrunds seitens der Kolonisatoren wird von Shakespeare als unerlässlich, letztendlich aber doch wirkungslos dargestellt. So kommt es, dass Prospero Caliban schließlich auf der niedrigsten sozialen Ebene ansiedelt, mit den Trunkenbolden und Straftätern Stephano und Trinculo gleichsetzt und ihn als hoffnungslos verlorenes „thing of darkness“ (V.i.275) [„Geschöpf der Finsternis“ (Bd. VI, 153)] bezeichnet:

Ein Teufel, ein geborner Teufel ist's,

An dessen Art Erziehung nimmer haftet,

An dem die Mühe, die ich menschlich nahm,

Ganz, ganz verloren ist, durchaus verloren;

Und wie sein Leib durchs Alter garst'ger wird,

Verstockt sein Sinn sich. ${ }^{56}$

$$
\text { (Bd. VI, 142) }
$$

Jener Prozess, mit Hilfe dessen Caliban zum Anderen gemacht wird, nämlich seine Unterwerfung und Behandlung als potentieller Krimineller (hier als Vergewaltiger und Rebell gegen alle Autorität) ist vor dem Hintergrund zeitgenössischer kultureller Ängste zu verstehen, vor allem im Kontext jener Spannungen, die aus dem Gegensatz von vermeintlicher Neue-Welt-Barbarei und europäischer Zivilisiertheit entstanden. Shakespeares Tempest ist damit ein weiteres Stück, in dem das Exotisch-Fremde scheinbar zur Unterhaltung des Publikums vorgeführt, sehr wahrscheinlich aber aus tiefer liegenden, vielleicht unbewussten Gründen eingesetzt wird, aus Gründen, die wohl mit Sorge und Zweifel hinsichtlich der eigenen kolonialen Aktivitäten in Zusammenhang stehen. Es dürfte deshalb nicht überraschen, dass auch in diesem Stück das Anderssein sehr schnell unterdrückt bzw. in Schach gehalten wird, vor allem dadurch, dass es als defizitär und minderwertig dargestellt wird.

56 „A devil, a born devil, on whose nature / Nurture can never stick; on whom my pains, / Humanely taken, all, all lost, quite lost; / And as with age his body uglier grows, / So his mind cankers.“" (The Tempest, IV.i.188-192) 


\section{Ergebnis}

Die bereits einleitend geäußerte Vermutung, es könne eine enge Verbindung geben zwischen Repräsentationen der Unterschiedlichkeit und dem kulturellen Bedürfnis, sich selbst zu definieren, lässt sich anhand der vier ausgewählten Texte bestätigen. In jedem dieser Stücke lassen sich Aspekte isolieren, die für das damalige Theaterpublikum von Relevanz gewesen sein dürften. Shakespeares Dramen entstanden zu einer Zeit, als zentrifugale Kräfte ihren Einfluss auf zeitgenössische Gesellschaften ausübten und damit widersprüchliche Reaktionen hervorriefen. Während die Europäer im Ausland nach neuen Märkten und Kolonien suchten und dabei als Spanier, Portugiesen und Engländer in die Welt expandierten, wurden sie selbst in vieler Hinsicht immer engstirniger und xenophober. Ihr Wissen um Macht, Reichtum und Gelehrsamkeit anderer Völker, aber auch ganz generell die Zunahme an Information über Kulturen außerhalb Europas, führte seltsamerweise verstärkt zum Ausdruck europäischer und christlicher Überlegenheit, was sich anhand von zeitgenössischen Diskursen nachweisen lässt. Gerade an der Darstellung von Unterschiedlichkeit, etwa in der Beschreibung von Mohren, Neue-Welt-Bewohnern und Juden im Theater der Frühen Neuzeit können wir sehen, wie sich eine Sprache entwickelte, mit deren Hilfe ein Europa im Wandel sich selbst neu zu definieren suchte. In England nahm der bereits bestehende Druck unter Elisabeth I. noch zu, zum einen durch die Angst vor einer Invasion, da die Regenten katholischer Völker die Rechtmäßigkeit von Elisabeths Herrschaft und damit ihre politische Position anzweifelten, zum anderen durch das Nachfolgeproblem mangels eines Tudorerben. Während Entdeckungsreisen die Erschließung neuer Handelsrouten sowie das Zusammentragen neuer Reichtümer in Aussicht stellten, rechtfertigte gerade England seine Expansionsbestrebungen in der Welt mit idealistischen Begründungen, unter anderem mit dem Export des wahren, nämlich protestantischen Glaubens, aber auch mit dem Verbreiten europäischer und deshalb zivilisierter Verhaltensformen in vermeintlich zurückgebliebene und barbarische Gebiete der Erde. Zugleich brachte das Bedürfnis nach Stabilität in einer Zeit des Wandels die Engländer dazu, ihre eigene Gesellschaft näher zu beleuchten und zwang sie, sich selbst Disziplin aufzuerlegen, dabei jenes zu unterdrücken, was zwar verlockend, aber verboten war, und schließlich ihr Selbstbild zu bestärken, indem sie andere als minderwertig und negativ einschätzten. Rückblickend erscheint deshalb dieses gleichzeitige Sichöffnen und Sichverschließen dem Anderen gegenüber als notwendiger Schritt, eine neue Mitte zu finden zu einer Zeit, in der, wie der Dichter John Donne es ausdrückte, die Welt „,in Trümmern war und aller Zusammenhalt weg“57 (Ü: BG). Zu dieser Zeit jedoch konnte noch niemand vorhersehen, dass dieser erste Schritt in die Welt England zu einem Kolonialreich verhelfen würde, von dem man 300 Jahre später sagen würde, dass die Sonne in ihm nie untergehe.

57 „Tis all in pieces, all coherence gone“, Donne, „First Anniversary“, Zeile 10. 


\section{Verwendete Literatur}

Primärwerke

Donne, John. „Elegy XIX: To His Mistress Going to Bed”. In: Seventeenth-Century Poetry: The Annotated Anthology. Ed. Terence Dawson und Robert Scott Dupree. Hemel Hempstead: Harvester Wheatsheaf, 1994. 38-42.

---. ,'The First Anniversary: An Anatomy of the World” (Ausschnitt). In: The New Oxford Book of Seventeenth Century Verse. Ed. Alastair Fowler. Oxford: Oxford University Press, 1991. 113/114.

Shakespeare, William. Antony and Cleopatra. [The Arden Shakespeare]. Ed. John Wilders. London: Routledge, 1995.

---. The Merchant of Venice. [The Arden Edition of the Works of William Shakespeare]. Ed. John Russell Brown. London: Methuen and Co Ltd, 1955.

---. Othello. [The Arden Edition of the Works of William Shakespeare]. Ed. M.R. Ridley. London: Methuen \& Co Ltd, 1969.

---. The Tempest. [The Arden Edition of the Works of William Shakespeare]. Ed. Frank Kermode. London: Methuen \& Co Ltd, 1954.

---. Sämtliche Werke. 6 Bde. Hg. Levin Schücking. Übersetzung von August Wilhelm Schlegel und Ludwig Tieck. Darmstadt: Tempel, 1955.

Wilmot, John. The Complete Poems of John Wilmot, Earl of Rochester. Ed. David M. Vieth. New Haven and London: Yale University Press, 1988.

Sekundärliteratur

Bartels, Emily C. „Making More of the Moor: Aaron, Othello, and Renaissance Refashionings of Race". Shakespeare Quarterly 41.4 (1990): 433-52.

Barthelemy, Anthony. Black Face Maligned Race: The Representation of Blacks in English Drama from Shakespeare to Southerne. Baton Rouge and London: Louisiana State University Press, 1987.

Boehmer, Elleke. Colonial and Postcolonial Literature: Migrant Metaphors. Oxford: Oxford University Press, 1995.

Cohwig, Ruth. „Blacks in English Renaissance Drama”. The Black Presence in English Literature. Ed. David Dabydeen. Manchester: Manchester University Press, 1985. 1-25.

D’Amico, Jack. The Moor in English Renaissance Drama. Tampa: University of South Florida Press, 1991.

Demaray, John G. Shakespeare and the Spectacles of Strangeness: The Tempest and the Transformation of Renaissance Theatrical Forms. Pittsburgh: Duquesne University Press, 1998. 
Jones, Eldred. Othello's Countrymen: The Africans in English Renaissance Drama. London: Oxford University Press, 1965.

Kristeva, Julia. Fremde sind wir uns selbst. (Etrangers à nous-mêmes). Übers. Xenia Rajewsky. Frankfurt: Suhrkamp, 1990.

Loomba, Ania. Gender, Race, Renaissance Drama. Manchester: Manchester University Press, 1989.

---. Shakespeare, Race, and Colonialism. Oxford: Oxford University Press, 2002.

Mannoni, Octave. Prospero and Caliban: The Psychology of Colonization. Trans. P. Powesland. London: Methuen, 1959.

Rosen, Alan. ,The Rhetoric of Exclusion: Jew, Moor, and the Boundaries of Discourse in The Merchant of Venice." Race, Ethnicity, and Power in the Renaissance. Ed. Joyce Green MacDonald. London: Associated United Presses, 1997. 67-79.

Said, Edward. Orientalism. London: Routledge and Kegan Paul, 1978.

Waddington, Raymond. „Rewriting the World, Rewriting the Body”. The Cambridge Companion to English Literature 1500-1600. Ed. Arthur F. Kinney. Cambridge: Cambridge University Press, 2000. 287-309.

Womack, Peter. „The Writing of Travel”. A Companion to English Renaissance Literature and Culture. Ed. Michael Hattaway. Oxford: Blackwell, 2000. 148-161. 